\title{
Algorithms for Solving Nonhomogeneous Generalized Sylvester Matrix Equations
}

\author{
Ehab A. El-Sayed $\mathbb{D}^{1,2}$ and Eid E. El Behady ${ }^{1}$ \\ ${ }^{1}$ Department of Mathematics, College of Science and Humanitarian Studies, Prince Sattam Bin Abdulaziz University, \\ Al-Kharj, Saudi Arabia \\ ${ }^{2}$ Department of Science and Mathematical Engineering, Faculty of Petroleum and Mining Engineering, Suez University, \\ Suez 43721, Egypt \\ Correspondence should be addressed to Ehab A. El-Sayed; ehab_math@yahoo.com
}

Received 24 December 2019; Revised 21 February 2020; Accepted 27 February 2020; Published 31 March 2020

Academic Editor: Filippo Cacace

Copyright ( $) 2020$ Ehab A. El-Sayed and Eid E. El Behady. This is an open access article distributed under the Creative Commons Attribution License, which permits unrestricted use, distribution, and reproduction in any medium, provided the original work is properly cited.

\begin{abstract}
This paper considers a new method to solve the first-order and second-order nonhomogeneous generalized Sylvester matrix equations $A V+B W=E V F+R$ and $M V F^{2}+D V F+K V=B W+R$, respectively, where $A, E, M, D, K, B$, and $F$ are the arbitrary real known matrices and $V$ and $W$ are the matrices to be determined. An explicit solution for these equations is proposed, based on the orthogonal reduction of the matrix $F$ to an upper Hessenberg form $H$. The technique is very simple and does not require the eigenvalues of matrix $F$ to be known. The proposed method is illustrated by numerical examples.
\end{abstract}

\section{Introduction}

Consider the following two homogeneous generalized Sylvester matrix equations:

$$
\begin{array}{r}
A V+B W=E V F, \\
M V F^{2}+D V F+K V=B W .
\end{array}
$$

Matrix equation (1) is called a first-order homogeneous generalized Sylvester matrix equation that is closely related to many problems in linear systems theory, such as eigenstructure assignment [1-5] and control of systems with input constraints [6]. Second-order homogeneous generalized Sylvester matrix equation (2) has found applications in many control problems, for example, pole assignment [7-9] and eigenstructure assignment $[10,11]$.

As a generalization of the above matrix equations, we have considered the following nonhomogeneous generalized Sylvester matrix equation:

$$
\begin{array}{r}
A V+B W=E V F+R, \\
M V F^{2}+D V F+K V=B W+R .
\end{array}
$$

where $A, E, M, D, K, B \in \mathbb{R}^{n \times n}, R \in \mathbb{R}^{n \times p}$, and $F \in \mathbb{R}^{p \times p}$ are the known matrices, while $V \in \mathbb{R}^{n \times p}$ and $W \in \mathbb{R}^{n \times p}$ need to be determined. Several authors have studied different methods for matrix equation (3) (see for example, Song and Chen, [12], Ramadan et al. [13], Duan [14], and Wu et al. [15]). The secondorder nonhomogeneous Sylvester matrix equation was introduced by Duan [16]. Recently, Ramadan et al. [17] developed the Hessenberg method to solve the Sylvester matrix equation $X A+B X=C$ by reducing only one coefficient matrix to a block upper Hessenberg form. The main goal of this paper is to present algorithms for solving well-known nonhomogeneous generalized Sylvester matrix equations (3) and (4). The proposed algorithm differs from the preceding standard methods; in our algorithms, $F$ is only reduced to an unreduced upper Hessenberg matrix $H$, and eigenvalues of matrix $F$ must be distinct, because if any eigenvalue of $H$ repeats, then it is 
defective. An unreduced Hessenberg matrix is always nonsingular, so $F$ must be nonsingular. Throughout this paper, the notation (GSME) is used for generalized Sylvester matrix equation and we assume that $\operatorname{det}(E) \neq 0, \operatorname{det}(F) \neq 0$, $\operatorname{det}(B) \neq 0$, and $\operatorname{det}(M) \neq 0$.

\section{The Proposed}

$$
\text { Method for } A V+B W=E V F+R
$$

Consider the following nonhomogeneous generalized firstorder Sylvester matrix equation:

$$
A V+B W=E V F+R,
$$

where $A, E, B \in \mathbb{R}^{n \times n}, R \in \mathbb{R}^{n \times p}$, and $F \in \mathbb{R}^{p \times p}$ are the known matrices, while $V, W \in \mathbb{R}^{n \times p}$ are to be determined. The following lemma plays a vital role in this paper.

Lemma 1 (see $[18,19])$. Let $H=\left(h_{i j}\right) \in \mathbb{R}^{p \times p}$ be an unreduced upper Hessenberg matrix and let $x_{1}, x_{2}, \ldots, x_{p}$ be the $p$ successive columns of a matrix $X$ that commutes with $H$; then,

(i) $x_{1}$ can be chosen arbitrarily.

(ii) The columns $x_{2}$ through $x_{p}$ can be computed recursively by the following formula:

$$
x_{i+1}=\frac{1}{h_{i+1, i}}\left(H x_{i}-\sum_{j=1}^{i} h_{j, i} x_{j}\right), \quad i=1,2, \ldots, p-1 .
$$

Algorithm 1 constructs an unknown matrix $L=\left\{l_{k}\right\}_{k=1}^{p} \in \mathbb{R}^{n \times p}$ and compute matrix $G=[\overline{0} \overline{0} \cdots g] \epsilon$ $\mathbb{R}^{n \times p}$ for the following matrix equation:

$$
A L+B G=E L H+S
$$

or where $l_{1}, l_{2}, \ldots, l_{p}$ are the columns of $\mathrm{L}, \overline{0}$ is the zero vector, $\mathrm{g}$ is the unknown vector, and $S=\left[s_{1}, s_{2}, \ldots, s_{p}\right]$ is the known real $n \times p$ matrix.

Theorem 1. The solution of matrix equation (5) is $V=L X Q^{T}$ and $W=G X Q^{T}$, where $A, E, B \in \mathbb{R}^{n \times n}, R \in \mathbb{R}^{n \times p}$, and $F \in \mathbb{R}^{p \times p}$ are the known matrices and the matrix $X$ is generated as in Lemma 1.

Proof. Matrix equation (5) can be rewritten in the following form:

$$
A V Q+B W Q=E V Q Q^{T} F Q+R Q
$$

That is,

$$
A \widehat{V}+B \widehat{W}=E \widehat{V} H+\widetilde{R},
$$

where $H=Q^{T} F Q \in \mathbb{R}^{p \times p}$ is an unreduced upper Hessenberg matrix, $\widetilde{R}=R Q$, and $Q \in \mathbb{R}^{p \times p}$ is an orthogonal similarity transformation. The matrix $X \in \mathbb{R}^{p \times p}$ is generated by (6), and matrix equation (7) is multiplied by $X$ to get

$$
A L X+B G X=E L H X+S X \text {. }
$$

By using Lemma $1(H X=X H)$, assume that $S X=\widetilde{R}, \widehat{V}=L X$, and $\widehat{W}=G X$, where $L$ and $G$ are computed from Algorithm 1. Then, we recover the original problem via the relations $V=\widehat{V} Q^{T}, W=\widehat{W} Q^{T}, R=\widetilde{R} Q^{T}$, and $F=Q H Q^{T}$, and then, the solution of (5) is $V=L X Q^{T}$ and $W=\mathrm{GXQ}^{T}$ (see Algorithm 2).

\section{The Proposed \\ Method of $M V F^{2}+D V F+K V=B W+R$}

Consider the following second-order nonhomogeneous generalized Sylvester matrix equation:

$$
M V F^{2}+D V F+K V=B W+R,
$$

where $M, D, K, B \in \mathbb{R}^{n \times n}, R \in \mathbb{R}^{n \times p}$, and $F \in \mathbb{R}^{p \times p}$ are the known matrices, while $V \in \mathbb{R}^{n \times p}$ and $W \in \mathbb{R}^{n \times p}$ are to be determined.

The following algorithm constructs an unknown matrix $L \in \mathbb{R}^{n \times p}$ and computes matrix $G \in \mathbb{R}^{n \times p}$ for the following matrix equation:

$$
M L H^{2}+D L H+K L=B G+S .
$$

Putting $U=L H$ in (13), we get

$$
M U H+D U+K L=B G+S,
$$

or 


\section{Step 1}

Choose $l_{1}$ arbitrarily

For $i=1,2, \ldots, p-1$ do

$l_{i+1}=\left(E^{-1} / h_{i+1, i}\right)\left(A l_{i}-s_{i}-\sum_{j=1}^{i} h_{j, i} E l_{j}\right)$;

Step 2

Compute $g$; for $B g=\left(s_{p}+\sum_{j=1}^{p} h_{j, p} E l_{j}-A l_{p}\right)$.

Algorithm 1: The proposed algorithm for $A L+B G=E L H+S$.

Input: Matrices $A, E, B \in \mathbb{R}^{n \times n}, R \in \mathbb{R}^{n \times p}$ and $F \in \mathbb{R}^{p \times p}$.

Output: Matrices $V$ and $W$.

Assumptions: $\operatorname{det}(E) \neq 0 \operatorname{det}(F) \neq 0, \operatorname{det}(B) \neq 0$, and $X$ are nonsingular matrix as shown in [19] and eigenvalues of matrix $F$ must be distinct.

Step 1: Reduce $F$ to an unreduced upper Hessenberg $H=Q^{T} F Q \in \mathbb{R}^{p \times p}$. Let $Q$ be an orthogonal matrix.

Step 2: Construct the matrix $X \in \mathbb{R}^{p \times p}$ generated by (6).

Step 3: Compute the matrix $S \in \mathbb{R}^{n \times p}$ by solving $S X=R Q$.

Step 4: Construct the matrices $\mathrm{L}$ and $\mathrm{G}$ as shown in Algorithm 1.

Step 5: Compute $V=L X Q^{T}$ and $W=G_{X Q}^{T}$.

Algorithm 2: The proposed algorithm for $A V+B W=E V F+R$.

$$
\begin{aligned}
& {\left[M u_{1}, M u_{2}, \ldots, M u_{p}\right]\left[\begin{array}{ccccc}
h_{11} & h_{12} & h_{13} & \cdots & h_{1 p} \\
h_{21} & h_{22} & h_{23} & \cdots & h_{2 p} \\
0 & h_{32} & h_{33} & \cdots & h_{3 p} \\
\vdots & \vdots & \vdots & \ddots & \vdots \\
0 & 0 & 0 & h_{p, p-1} & h_{p p}
\end{array}\right]+\left[D u_{1}, D u_{2}, \ldots, D u_{p}\right]+\left[K l_{1}, K l_{2}, \ldots, K l_{p}\right]=\left[\overline{0}, \overline{0}, \ldots, \overline{0}, B g_{1}, B g_{2}\right]} \\
& +\left[s_{1}, s_{2}, \ldots, s_{p}\right] .
\end{aligned}
$$

Since $U=L H$, then

$$
u_{i}=\sum_{j=1}^{i+1} h_{j, i} l_{j}, \quad \text { for } i=1,2, \ldots, p-1 .
$$

Both $\quad\left\{l_{1}, l_{2}, \ldots, l_{p}\right\}, \quad\left\{u_{1}, u_{2}, \ldots, u_{p}\right\}, \quad$ and $\left\{s_{1}, s_{2}, \ldots, s_{p}\right\}$ are columns of $L, U$, and $S$, respectively. $G=$ $\left[\overline{0}, \overline{0}, \ldots, \overline{0}, g_{1}, g_{2}\right]$ is a $n \times p$ real matrix, where $\overline{0}$ is the zero vector and $g_{1}$ and $g_{2}$ are the unknown vectors. From (15) and (16), we design Algorithm 3.

Theorem 2. The solution of matrix equation (12) is $V=$ $L X Q^{T}$ and $W=G X Q^{T}$, where $M, D, K, B \in \mathbb{R}^{n \times n}, R \in$ $\mathbb{R}^{n \times p}$, and $F \in \mathbb{R}^{p \times p}$ are the known matrices, and the matrix $X$ is generated as in Lemma 1.

Proof. Matrix equation (12) can be rewritten in the Hessenberg form as follows:

$$
M V Q Q^{T} F Q Q^{T} F Q+D V Q Q^{T} F Q+K V Q=B W Q+R Q .
$$

That is

$$
M \widehat{V} H^{2}+D \widehat{V} H+K \widehat{V}=B \widehat{W}+\widetilde{R},
$$

where $\widehat{W}=W Q$ and $\widehat{V}=V Q$ are to be determined, while $H=Q^{T} F Q \in \mathbb{R}^{p \times p}$ is an unreduced upper Hessenberg matrix, $\widetilde{R}=R Q$, and $Q \in \mathbb{R}^{p \times p}$ is an orthogonal similarity transformation. The main idea is to find a matrix $L$ and matrix $G$ for a new matrix equation (13) as shown in Algorithm 3, where $S=\widetilde{R} X^{-1}$ is known as $\mathrm{n} \times \mathrm{p}$ real matrix while the matrix $X \in \mathbb{R}^{p \times p}$ is generated by (6). Multiply matrix equation (13) by $X$; then,

$$
M L H^{2} \mathrm{X}+D L H \mathrm{X}+K L X=B G X+S \mathrm{X} .
$$

By using Lemma 1 and assuming that $\widehat{V}=L \mathrm{X}, \widehat{W}=G \mathrm{X}$, and $S=\widetilde{R} X^{-1}$ we have

$$
M \widehat{V} H^{2}+D \widehat{V} H+K \widehat{V}=B \widehat{W}+\widetilde{R} .
$$




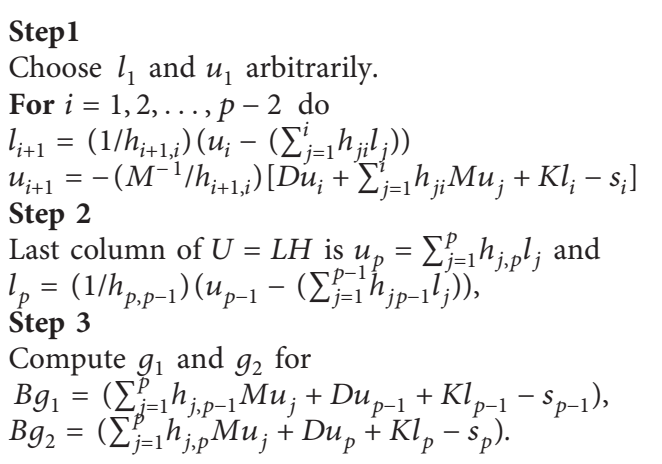

Algorithm 3: The proposed algorithm for $M L H^{2}+D L H+K L=B G+\mathrm{S}$.

Input: Matrices $M, \quad D, K, B \in \mathbb{R}^{n \times n}, R \in \mathbb{R}^{n \times p}$ and $\quad F \in \mathbb{R}^{p \times p}$.

Output: Matrices $V$ and $W$.

Assumptions: de $t(M) \neq 0$, de $t(F) \neq 0$, de $\quad t(B) \neq 0$ and $X$ is nonsingular matrix as shown in [19] and eigenvalues of matrix $F$ must be distinct.

Step 1: Reduce $F$ to an unreduced upper Hessenberg $H=Q^{T} F Q \in \mathbb{R}^{p \times p}$. Let $Q$ be an orthogonal matrix.

Step 2: Construct the matrix $X \in \mathbb{R}^{p \times p}$ generated by (6).

Step 3: Compute the matrix $S \in \mathbb{R}^{n \times p}$ by solving $S X=R Q$.

Step 4: Construct the matrices $\mathrm{L}$ and $\mathrm{G}$ as shown in Algorithm 3.

Step 5: Compute $V=L X Q^{T}$ and $W=G X^{T}$.

Algorithm 4: The proposed algorithm for $M V F^{2}+D V F+K V=B W+R$.

We then recover the original problem via the relations $V=\widehat{V} Q^{T}, W=\widehat{W} Q^{T}$, and $F=Q H Q^{T}$; then, the solution of (12) is $V=L X Q^{T}$ and $W=G X Q^{T}$ (see Algorithm 4).

\section{Numerical Examples}

In this section, we present two numerical examples to illustrate the application of our proposed method.

Example 1. We solve first-order GSME (3) where $A, E, B \in R^{n \times n}$ and $F \in R^{p \times p}$ are random matrices. The accuracy of the proposed method is reported for different values of $n$, when $p=2$, and for different values of $p$, when $n=10$, as in Table 1 .

Example 2. We solve the second-order GSME (4) where $M, D, K, B \in R^{n \times n}$ and $F \in R^{p \times p}$ are random matrices. The accuracy of the proposed method is reported for different values of $n$, when $p=2$, and for different values of $p$, when $n=10$, as in Table 2 .

\section{Discussion}

The accuracy of the proposed methods is remarkably dependent on $p$. If $p$ is too large, one of the entries $h_{i+1, i}$ of
TABLE 1: The accuracy error for Algorithm 2.

\begin{tabular}{lccc}
\hline$n$ & $\|A V+B W-E V F-R\|_{2}$ & $p$ & $\|A V+B W-E V F-R\|_{2}$ \\
\hline 6 & $1.2437 \times 10^{-14}$ & 2 & $8.0572 \times 10^{-15}$ \\
10 & $8.0572 \times 10^{-15}$ & 4 & $3.7550 \times 10^{-12}$ \\
100 & $2.2609 \times 10^{-12}$ & 6 & $1.5518 \times 10^{-9}$ \\
1000 & $2.8632 \times 10^{-9}$ & 8 & $1.5072 \times 10^{-5}$ \\
\hline
\end{tabular}

$H$ may have a tendency to be zero, which affects singularity of the $X$ matrix. Thus, the smaller the value of $p$, the greater the precision. In previous studies of GSME $A V+$ $B W=E V F+R[12-15]$ and $M V F^{2}+D V F+K V=B W+$ $R$ [16], the working hypotheses that size $n$ of known matrices are very small. However, in our method, $n$ is very large.

\section{Conclusion}

In this study, the solution of GSME $A V+B W=E V F+R$ and $M V F^{2}+D V F+K V=B W+R$, where $A, B, E, M, D, K, R$, and $F$ are arbitrary real known matrices and $V$ and $W$ are the matrices to be determined, is investigated. With the help of orthogonal similarity transformation and reduction to Hessenberg form, some good results are obtained. The 
TABle 2: The accuracy error for Algorithm 4.

\begin{tabular}{lccc}
\hline$n$ & $\left\|M V F^{2}+D V F+K V-B W-R\right\|_{2}$ & $p$ & $\left\|M V F^{2}+D V F+K V-B W-R\right\|_{2}$ \\
\hline 6 & $4.8449 \times 10^{-15}$ & 2 & $9.9654 \times 10^{-15}$ \\
10 & $9.9654 \times 10^{-15}$ & 4 & $8.6627 \times 10^{-13}$ \\
100 & $1.0817 \times 10^{-12}$ & 6 & $4.1397 \times 10^{-11}$ \\
1000 & $1.0817 \times 10^{-12}$ & 8 & $4.0780 \times 10^{-8}$ \\
\hline
\end{tabular}

proposed techniques are tested by solving two test problems where the accuracy is seen to be highly remarkable.

\section{Open Problem}

Extend the Hessenberg method to solve Sylvester quaternion matrix equation [20] and coupled matrix equation [21].

\section{Data Availability}

The data used to support the findings of this study are included within the article.

\section{Conflicts of Interest}

The authors declare that they have no conflicts of interest.

\section{Acknowledgments}

This project was supported by the Deanship of Scientific Research at Prince Sattam Bin Abdulaziz University under the research project \#2017/01/7944. The authors are very grateful to Professor Reny George for the proofreading of the research.

\section{References}

[1] G. R. Duan, "Solution to matrix equation AV + BW =EVF and eigenstructure assignment for descriptor systems," Automatica, vol. 28, no. 3, pp. 639-642, 1992.

[2] G.-R. Duan, "Solutions of the equation $\mathrm{AV}+\mathrm{BW}=\mathrm{VF}$ and their application to eigenstructure assignment in linear systems," IEEE Transactions on Automatic Control, vol. 38, no. 2, pp. 276-280, 1993.

[3] G.-R. Duan, "Eigenstructure assignment in descriptor systems via output feedback: a new complete parametric approach," International Journal of Control, vol. 72, no. 4, pp. 345-364, 1999.

[4] L. Fletcher, J. Kautsky, and N. Nichols, "Eigenstructure assignment in descriptor systems," IEEE Transactions on Automatic Control, vol. 31, no. 12, pp. 1138-1141, 1986.

[5] F. L. Lewis and K. Ozcaldiran, "Geometric structure and feedback in singular systems," IEEE Transactions on Automatic Control, vol. 34, no. 4, pp. 450-455, 1989.

[6] A. Saberi, A. A. Stoorvogel, and P. Sannuti, Control of Linear Systems with Regulation and Input Constraints, Springer Science \& Business Media, Berlin, German, 2012.

[7] Y. Kim, H.-S. Kim, and J. L. Junkins, "Eigenstructure assignment algorithm for mechanical second-order systems," Journal of Guidance, Control, and Dynamics, vol. 22, no. 5, pp. 729-731, 1999.

[8] E. K. Chu and B. N. Datta, "Numerically robust pole assignment for second-order systems," International Journal of Control, vol. 64, no. 6, pp. 1113-1127, 1996.
[9] M. Dehghan and M. Hajarian, "Efficient iterative method for solving the second-order Sylvester matrix equation $\mathrm{EVF}_{2}-\mathrm{AVF}-\mathrm{CV}=\mathrm{BW}$, IET Control Theory \& Applications, vol. 3, no. 10, pp. 1401-1408, 2009.

[10] G. Duan, "Two parametric approaches for eigenstructure assignment in second-order linear systems," Journal of Control Theory and Applications, vol. 1, no. 1, pp. 59-64, 2003.

[11] G.-R. Duan and B. Zhou, "Solution to the second-order sylvester matrix equation," IEEE Transactions on Automatic Control, vol. 51, no. 5, pp. 805-809, 2006.

[12] C. Song and G. Chen, "Solutions to matrix equations $\mathrm{X}-\mathrm{AXB}=\mathrm{CY}+\mathrm{R}$ and $\mathrm{X}-\mathrm{AXB}=\mathrm{CY}+\mathrm{R}$," Journal of Computational and Applied Mathematics, vol. 343, pp. 488-500, 2018.

[13] M. A. Ramadan, N. M. El-Shazly, and B. I. Selim, "The antireflexive solutions for the matrix equation $\mathrm{AV}+\mathrm{BW}=\mathrm{EVF}+\mathrm{C}, "$ Computational and Applied Mathematics, vol. 38, no. 2, p. 86, 2019.

[14] G.-R. Duan, "The solution to the matrix equation AV + BW $=\mathrm{EVJ}+\mathrm{R}$," Applied Mathematics Letters, vol. 17, no. 10, pp. 1197-1202, 2004.

[15] A.-G. Wu, Y. Sun, and G. Feng, "Closed-form solution to the non-homogeneous generalised Sylvester matrix equation," IET Control Theory \& Applications, vol. 4, no. 10, pp. 19141921, 2010.

[16] G.-R. Duan, "Solution to second-order nonhomogeneous generalized Sylvester equations," in Proceedings of the 2013 9th Asian Control Conference (ASCC), IEEE, Guiyang, China, May 2013.

[17] M. A. Ramadan, N. M. El-Shazly, and B. I. Selim, "A Hessenberg method for the numerical solutions to types of block Sylvester matrix equations," Mathematical and Computer Modelling, vol. 52, no. 9-10, pp. 1716-1727, 2010.

[18] B. Datta and K. Datta, "On eigenvalue and canonical form assignments," Linear Algebra and Its Applications, vol. 131, pp. 161-182, 1990.

[19] M. A. Ramadan and E. A. El-Sayed, "On the matrix equation $\mathrm{XH}=\mathrm{HX}$ and the associated controllability problem," Applied Mathematics and Computation, vol. 186, no. 1, pp. 844-859, 2007.

[20] I. Kyrchei, "Cramer's rules for Sylvester quaternion matrix equation and its special cases," Advances in Applied Clifford Algebras, vol. 28, no. 5, p. 90, 2018.

[21] M. Hajarian, "Convergence analysis of the MCGNR algorithm for the least squares solution group of discrete-time periodic coupled matrix equations," Transactions of the Institute of Measurement and Control, vol. 39, no. 1, pp. 29-42, 2017. 